\title{
Sex-Specific Association between Serum Vitamin D Status and Lipid Profiles: A Cross-Sectional Study of a Middle-Aged and Elderly Chinese Population
}

\author{
Fei HuANG ${ }^{1}$, Qingquan $\mathrm{LIU}^{1}$, Qian ZHANG ${ }^{1}$, Zhengce WAN ${ }^{2}$, Liu Hu' ${ }^{2}$, \\ Ranran $\mathrm{Xu}^{1}$, Anying $\mathrm{CHENG}^{1}$, Yongman $\mathrm{LV}^{1,2}$ and Le WANG ${ }^{1, *}$ \\ ${ }^{1}$ Department of Nephrology, and ${ }^{2}$ Department of Health Management Centre, Tongji Hospital, \\ Tongji Medical College, Huazhong University of Science and Technology, Wuhan, China
}

(Received September 22, 2019)

\begin{abstract}
Summary Studies have shown that vitamin D status might be associated with dyslipidaemia, but results are conflicting and there might exist sex differences. The aim of our study was to explore the sex-specific association between vitamin D status and serum lipids and atherogenic index of plasma (AIP, a predictor for atherosclerosis) among Chinese middleaged and elderly adults. A total of 4,021 middle-aged and elderly participants from a health management centre were included in this cross-sectional study. The individuals were classified into tertiles according to serum 25(OH)D. Linear and logistic regression models were used to estimate the association between vitamin D levels and serum lipids among the tertiles. The mean serum 25(OH)D level was $21.60(16.60-27.20) \mathrm{ng} / \mathrm{mL}$ in all participants. After adjusting for potential confounders, a $10 \mathrm{ng} / \mathrm{mL}$ increase in $25(\mathrm{OH}) \mathrm{D}$ was associated with decreases of $1.156 \mathrm{mmol} / \mathrm{L}$ in triglycerides (TGs) and 0.068 in the AIP and an increase of $0.051 \mathrm{mmol} / \mathrm{L}$ in high-density lipoprotein cholesterol (HDL-C) in all subjects. In addition, 25(OH)D deficiency was associated with an increased prevalence of hypertriglyceridaemia (odds ratio (OR), 1.880; 95\% confidence interval (CI), 1.351-2.615), hypoalphalipoproteinaemia/HDL (OR, 1.505; 95\% CI, 1.146-1.977) and abnormal AIP (OR, 1.933; 95\% CI, 1.474-2.534) in males, and 25(OH)D-deficient women had a 2.02 -fold higher risk for hypoalphalipoproteinaemia/HDL than women with sufficient $25(\mathrm{OH}) \mathrm{D}$ levels $(95 \% \mathrm{CI}$, 1.044-3.904; all $p$ values $<0.05$ ). Vitamin D deficiency was positively associated with the prevalence of dyslipidaemia and abnormal AIP in the middle-aged and elderly Chinese population. And this association was stronger in men than in women.
\end{abstract}

Key Words vitamin D, atherogenic index of plasma, dyslipidaemia, sex difference, gerontology

Vitamin D is an essential endogenous secosteroid hormone with a multitude of functions (1). The predominant source of vitamin D is solar ultraviolet (UV) radiation, which stimulates the transformation of 7-dehydrocholesterol to previtamin $\mathrm{D}_{3}$ in the skin. Previtamin $\mathrm{D}_{3}$ is continuously converted to vitamin $\mathrm{D}_{3}$ with thermal isomerization by body temperature. Vitamin D, including vitamin $\mathrm{D}_{3}$ and vitamin $\mathrm{D}_{2}$, can be sequentially hydroxylated into 25-hydroxyvitamin D [25(OH)D] and 1,25-dihydroxyvitamin $\mathrm{D}\left[1,25(\mathrm{OH})_{2} \mathrm{D}\right]$ in the liver and the kidney (2). Currently, the vitamin D status of the body is mainly evaluated by measuring $25(\mathrm{OH}) \mathrm{D}(3)$. In addition to the traditional functions of maintaining calcium homeostasis and bone metabolism, vitamin D has been reported to be associated with a broad range of chronic conditions, such as metabolic syndrome, diabetes, and autoimmune diseases (4-6). Cardiovascular diseases (CVDs) are among the most prevalent conditions linked to vitamin D deficiency (7). Given that middle-

\footnotetext{
*To whom correspondence should be addressed.
}

E-mail: lwang1@foxmail.com aged and elderly people are especially at risk of vitamin D deficiency and CVD (8), we focused on mainly this target population.

CVD is one of the main causes of death around the world, and there is accumulating evidence indicating an association between low levels of $25(\mathrm{OH}) \mathrm{D}$ and increased risk of CVD and all-cause mortality $(7,9)$. Disorders of lipid profiles (dyslipidaemia), including increased levels of total cholesterol (TC), triglycerides (TGs) and low-density lipoprotein cholesterol (LDL-C) and decreased levels of high-density lipoprotein cholesterol (HDL-C), have been identified as important contributing factors in atherosclerosis and CVD (10). Previous studies have reported a correlation between $25(\mathrm{OH}) \mathrm{D}$ levels and serum lipids $(11,12)$. However, the association between vitamin $\mathrm{D}$ and lipid profiles is divergent and inconclusive in the different ethnic groups that have been studied (13, 14). In addition, these inconsistencies might be caused by sex differences. Some studies have demonstrated that postmenopausal women with vitamin D deficiency had a higher risk of hypertriglyceridaemia and low HDL-C concentrations than women with sufficient vitamin D 
levels $(4,15)$. However, several studies of the general population have shown that vitamin $\mathrm{D}$ deficiency could influence men's serum lipid profiles more than those of women $(16,17)$. These studies have investigated only one sex or used a sample of adults rather than limiting the analyses to a middle-aged and elderly population, so data on sex differences in the association is sparse, especially from Asian populations.

Therefore, the main purpose of this study was to investigate the independent association between vitamin D and blood lipids and to show potential differences by sex in a representative sample of urban, middle-aged and elderly adults residing in central China. The atherogenic index of plasma (AIP), an index composed of TGs and HDL-C that has been reported to quantify blood lipid levels and that is used as an indicator of dyslipidaemia and CVD $(18,19)$, was also investigated in our study.

\section{MATERIALS AND METHODS}

Participants. The present study was a cross-sectional study that included participants aged $45-85$ y from the health management centre in the Optics Valley branch of Tongji Hospital (Wuhan, China). Data were collected between January 2017 and December 2017. Initially, 4,937 individuals were identified as potential participants. Subjects were excluded for the following reasons: (1) past history of severe liver or kidney diseases, thyroid or parathyroid disorders, cardiovascular disease or diabetes $(n=408)$; (2) use of vitamin D and calcium supplementation, use of medications that affect vitamin $\mathrm{D}$ and lipid profiles (i.e., lipid-lowering drugs) $(n=39)$ and (3) missing data for any of the study variables $(n=469)$. Finally, a total of 4,021 eligible participants (2,077 males and 1,944 females) were included for our analysis.

The study was approved by the Ethics Committee of Tongji Hospital, Tongji Medical College, Huazhong University of Science and Technology (The Institutional Review Board Approval Number: TJ-C20160115). The study conforms to the principles outlined in the Declaration of Helsinki and written informed consent was obtained from all participants.

Anthropometric and biochemical measurements. Routine anthropometric measurements were performed by trained examiners. Height and weight were measured to the nearest $0.1 \mathrm{~cm}$ and $0.1 \mathrm{~kg}$, respectively, while the participants wore light clothing and stood barefoot. Body mass index (BMI) was calculated as weight $(\mathrm{kg}) /$ height $(\mathrm{m})^{2}$. After resting quietly for a minimum of $5 \mathrm{~min}$, systolic blood pressure (SBP) and diastolic blood pressure (DBP) were measured using a standard electronic sphygmomanometer (HBP-9020; OMRON, Dalian, China). Data on sex, age, ethnicity and history of disease were obtained by using a questionnaire. Fasting (over $12 \mathrm{~h}$ ) blood samples were collected from each participant and then sent to the clinical laboratory of Tongji Hospital for standard biochemical measurements, including TC, TGs, LDL-C, HDL-C, fasting plasma glucose (FPG), glycosylated haemoglobin (HbA1c), uric acid, creatinine, C-reactive protein (CRP), homocysteine
(Hcy), calcium and phosphate. Serum levels of 25(OH)D were determined using the LIAISON ${ }^{\circledR} 25-\mathrm{OH}$ Vitamin D Total assay (DiaSorin, Inc., Stillwater, MN, USA) following the manufacturer's instructions.

Criteria and definitions. According to the commonly accepted cut off (20), vitamin D status was categorized as deficient $(<20 \mathrm{ng} / \mathrm{mL})$, insufficient $(20-30 \mathrm{ng} / \mathrm{mL})$, or sufficient ( $>30 \mathrm{ng} / \mathrm{mL}$ ). On that basis, we divided the 2,077 male participants and 1,944 female participants into three tertiles. Tertile 1 (T1) had the lowest mean 25(OH)D concentration, and Tertile 3 (T3) had the highest mean $25(\mathrm{OH}) \mathrm{D}$ concentration. In our study, the cutoff values for high TC, high TGs, high LDL-C and low HDL-C were $6.22 \mathrm{mmol} / \mathrm{L}, 2.26 \mathrm{mmol} / \mathrm{L}, 4.14 \mathrm{mmol} / \mathrm{L}$ and $1.04 \mathrm{mmol} / \mathrm{L}$, respectively (21). The AIP, calculated as the logarithmically transformed ratio of TGs to HDL-C $(\log [\mathrm{TGs} / \mathrm{HDL}-\mathrm{C}])$, is thought to be a useful independent predictor of CVD. An AIP $>0.15$ was regarded as abnormal in this study (22).

Statistical analysis. Continuous variables are presented as the means \pm standard deviations (SDs), nonnormally distributed continuous variables are expressed as medians (25th-75th) and categorical variables are expressed as frequencies. Sex differences were examined using independent $t$-tests and chi-square tests. Across the 25(OH)D tertiles, comparisons of the groups were performed using one-way ANOVA for continuous variables, the Kruskal-Wallis test for non-normally distributed variables and the chi-square test for categorical variables. Multivariate linear regression models were used to examine the association between serum 25(OH)D concentrations and lipid status. Some variables were logarithmically transformed prior to being analyzed. Logistic regression models were used to determine the relationship between $25(\mathrm{OH}) \mathrm{D}$ deficiency and the prevalence of dyslipidaemia, with data presented as odds ratios (ORs) and 95\% confidence intervals (CIs). In this study, we used 2 models to adjust potential confounders of dyslipidaemia. Model 1 was adjusted for age, SBP, DBP, fasting blood glucose (FBG), uric acid and creatinine. Model 2 included the variables in Model 1 and BMI. We conducted subgroup analyses to explore the association between vitamin D deficiency and dyslipidaemia according to BMI $\left(<24\right.$ or $\geq 24 \mathrm{~kg} / \mathrm{m}^{2}$, the cutoff value of overweight for Asian individuals) and age ( $<53$ or $\geq 53 \mathrm{y}$, the median age of the participants). We conducted tests for interactions among the subgroups using the Wald test. All statistical analyses were performed using SPSS software version 17.0 (SPSS Inc., Chicago, IL, USA). Two-tailed $p$ values of $<0.05$ were considered statistically significant.

\section{RESULTS}

\section{General characteristics of the participants}

A total of 4,021 subjects were included in the study, and $51.7 \%$ of the subjects were male. The mean age of the study population was $55.53 \mathrm{y}$. The mean serum $25(\mathrm{OH}) \mathrm{D}$ concentration was $21.60 \mathrm{ng} / \mathrm{mL}$. The incidence rates of vitamin $\mathrm{D}$ deficiency and insufficiency were $42.0 \%$ and $41.5 \%$, respectively. Table 1 shows the 
Table 1. Characteristics of subjects.

\begin{tabular}{|c|c|c|c|c|}
\hline & All & Male & Female & $\mathrm{M} / \mathrm{F} p$ value \\
\hline$n$ & 4,021 & 2,077 & 1,944 & \\
\hline Age (y) & $55.53 \pm 8.96$ & $54.30 \pm 8.07$ & $56.83 \pm 9.65$ & $<0.001$ \\
\hline BMI $\left(\mathrm{kg} / \mathrm{m}^{2}\right)$ & $24.18 \pm 2.99$ & $24.82 \pm 2.90$ & $23.50 \pm 2.94$ & $<0.001$ \\
\hline SBP (mmHg) & $129.45 \pm 18.99$ & $130.58 \pm 18.10$ & $128.25 \pm 19.84$ & $<0.001$ \\
\hline DBP (mmHg) & $77.31 \pm 12.25$ & $80.37 \pm 11.95$ & $74.04 \pm 11.72$ & $<0.001$ \\
\hline $\mathrm{TC}(\mathrm{mmol} / \mathrm{L})$ & $4.76 \pm 0.87$ & $4.70 \pm 0.86$ & $4.83 \pm 0.88$ & $<0.001$ \\
\hline TGs $(\mathrm{mmol} / \mathrm{L})$ & $1.62 \pm 1.28$ & $1.84 \pm 1.54$ & $1.38 \pm 0.86$ & $<0.001$ \\
\hline LDL-C (mmol/L) & $2.89 \pm 0.77$ & $2.90 \pm 0.77$ & $2.88 \pm 0.77$ & 0.354 \\
\hline HDL-C (mmol/L) & $1.31 \pm 0.32$ & $1.19 \pm 0.27$ & $1.44 \pm 0.32$ & $<0.001$ \\
\hline AIP & $0.03 \pm 0.31$ & $0.12 \pm 0.31$ & $-0.06 \pm 0.27$ & $<0.001$ \\
\hline FBG (mmol/L) & $5.48 \pm 1.02$ & $5.57 \pm 1.16$ & $5.38 \pm 0.84$ & $<0.001$ \\
\hline $\mathrm{HbA1c}(\%)$ & $5.69 \pm 0.63$ & $5.70 \pm 0.69$ & $5.68 \pm 0.54$ & 0.672 \\
\hline Uric acid $(\mu \mathrm{mol} / \mathrm{L})$ & $337.08 \pm 89.91$ & $384.40 \pm 82.42$ & $286.47 \pm 67.14$ & $<0.001$ \\
\hline Creatinine $(\mu \mathrm{mol} / \mathrm{L})$ & $74.15 \pm 16.40$ & $84.62 \pm 13.90$ & $62.94 \pm 10.36$ & $<0.001$ \\
\hline $\mathrm{CRP}(\mathrm{mg} / \mathrm{L})$ & $2.24 \pm 7.54$ & $2.29 \pm 6.99$ & $2.15 \pm 8.45$ & 0.794 \\
\hline Hcy $(\mu \mathrm{mol} / \mathrm{L})$ & $13.79 \pm 7.56$ & $16.29 \pm 8.95$ & $11.08 \pm 4.26$ & $<0.001$ \\
\hline $\mathrm{Ca}(\mathrm{mmol} / \mathrm{L})$ & $2.34 \pm 0.08$ & $2.34 \pm 0.08$ & $2.34 \pm 0.09$ & 0.848 \\
\hline $\mathrm{P}(\mathrm{mmol} / \mathrm{L})$ & $1.02 \pm 0.16$ & $0.96 \pm 0.14$ & $1.11 \pm 0.14$ & $<0.001$ \\
\hline $25(\mathrm{OH}) \mathrm{D}(\mathrm{ng} / \mathrm{mL})$ & $21.60(16.60-27.20)$ & $23.10(18.00-28.70)$ & $20.00(15.40-25.60)$ & $<0.001$ \\
\hline Dyslipidaemia, $n(\%)$ & $1,360(33.8)$ & $954(45.9)$ & $406(20.9)$ & $<0.001$ \\
\hline
\end{tabular}

Data are presented as the mean \pm SD, median (25th-75th) or percentages.

BMI, body mass index; SBP, systolic blood pressure; DBP, diastolic blood pressure; TC, total cholesterol; TGs, triglycerides; LDL-C, low-density lipoprotein cholesterol; HDL-C, high-density lipoprotein cholesterol; AIP, atherogenic index of plasma; FBG, fasting blood glucose; HbA1c, glycosylated haemoglobin; CRP, C-reactive protein; Hcy, homocysteine; Ca, calcium; P, phosphate.

general characteristics of the research subjects according to sex. The female participants were older; had a lower BMI; had higher levels of TC and HDL-C; had lower levels of blood pressure, TGs, AIP, FPG, Hcy, uric acid, creatinine, and 25(OH)D; and had a higher prevalence of dyslipidaemia (all $p$ values $<0.05$ ).

To further investigate the characteristic differences, we divided the men and women into tertiles for comparison. The incidence of vitamin D deficiency was $34.5 \%$ and $49.9 \%$ in males and females, respectively. In males, we found significantly lower levels of TC, TGs, BMI, and AIP; a higher level of HDL-C; and a lower prevalence of dyslipidaemia in the third tertile compared with the values of the participants in the first and second tertiles (all $p$ values $<0.05)$. However, in female participants, there were significant differences in BMI, HDL-C, uric acid, creatinine, calcium and phosphate among the three tertiles (Table 2).

Association between serum 25(OH)D concentrations and serum lipids

Multiple linear regression analyses were performed to assess the relationship between $25(\mathrm{OH}) \mathrm{D}$ levels and lipid profiles. In all subjects, $25(\mathrm{OH}) \mathrm{D}$ concentrations were negatively associated with TGs and AIP and positively associated with HDL-C after adjusting for age, SBP, DBP, FBG, uric acid, and creatinine (Model 1). Further adjustment for BMI (Model 2) did not substantially alter the associations. Our results showed that each $10 \mathrm{ng} / \mathrm{mL}$ increase in 25(OH)D was associated with decreases of $1.156 \mathrm{mmol} / \mathrm{L}$ in TGs and $0.068 \mathrm{in}$ AIP and an increase of $0.051 \mathrm{mmol} / \mathrm{L}$ in HDL-C (all $p$ values $<0.05$ ). However, TC and LDL-C showed no significant linear relationship with 25(OH)D (Table 3).

Moreover, we found negative associations between 25(OH)D and TC, TGs and AIP and a positive association between 25(OH)D and HDL-C in males after adjusting for the confounders in Model 1 and Model 2. A $10 \mathrm{ng} / \mathrm{mL}$ increase in $25(\mathrm{OH}) \mathrm{D}$ was associated with decreases of $1.279 \mathrm{mmol} / \mathrm{L}$ in TGs, $0.047 \mathrm{mmol} / \mathrm{L}$ in TC, and 0.119 in AIP and an increase of $0.102 \mathrm{mmol} / \mathrm{L}$ in HDL-C (all $p$ values $<0.05)$. In contrast, in female participants, there was a positive association between only $25(\mathrm{OH}) \mathrm{D}$ and HDL-C in Model 1 and Model 2 (Table 4). Each $10 \mathrm{ng} / \mathrm{mL}$ increase in $25(\mathrm{OH}) \mathrm{D}$ was associated with an increase of $0.060 \mathrm{mmol} / \mathrm{L}$ in HDL-C $(p=0.006)$.

Relationship between serum 25(OH)D concentrations and the occurrence of dyslipidaemia

The ORs and 95\% CIs for dyslipidaemia according to serum $25(\mathrm{OH}) \mathrm{D}$ concentrations in men and women are presented in Table 5. The OR (95\% CI) for hypertriglyceridaemia, hypoalphalipoproteinaemia and abnormal AIP increased notably in vitamin D-deficient men compared to the corresponding ORs of vitamin D-sufficient men after adjustment for age, hypertension, FBG, uric acid, and creatinine. The relationship between serum 25(OH)D concentrations and the occurrences of hypertriglyceridaemia, hypoalphalipoproteinaemia and abnormal AIP remained significant after additional adjustment for BMI, with ORs and 95\% CIs of 1.880 (1.351-2.615), 1.505 (1.146-1.977), and 1.933 (1.474-2.534), re- 


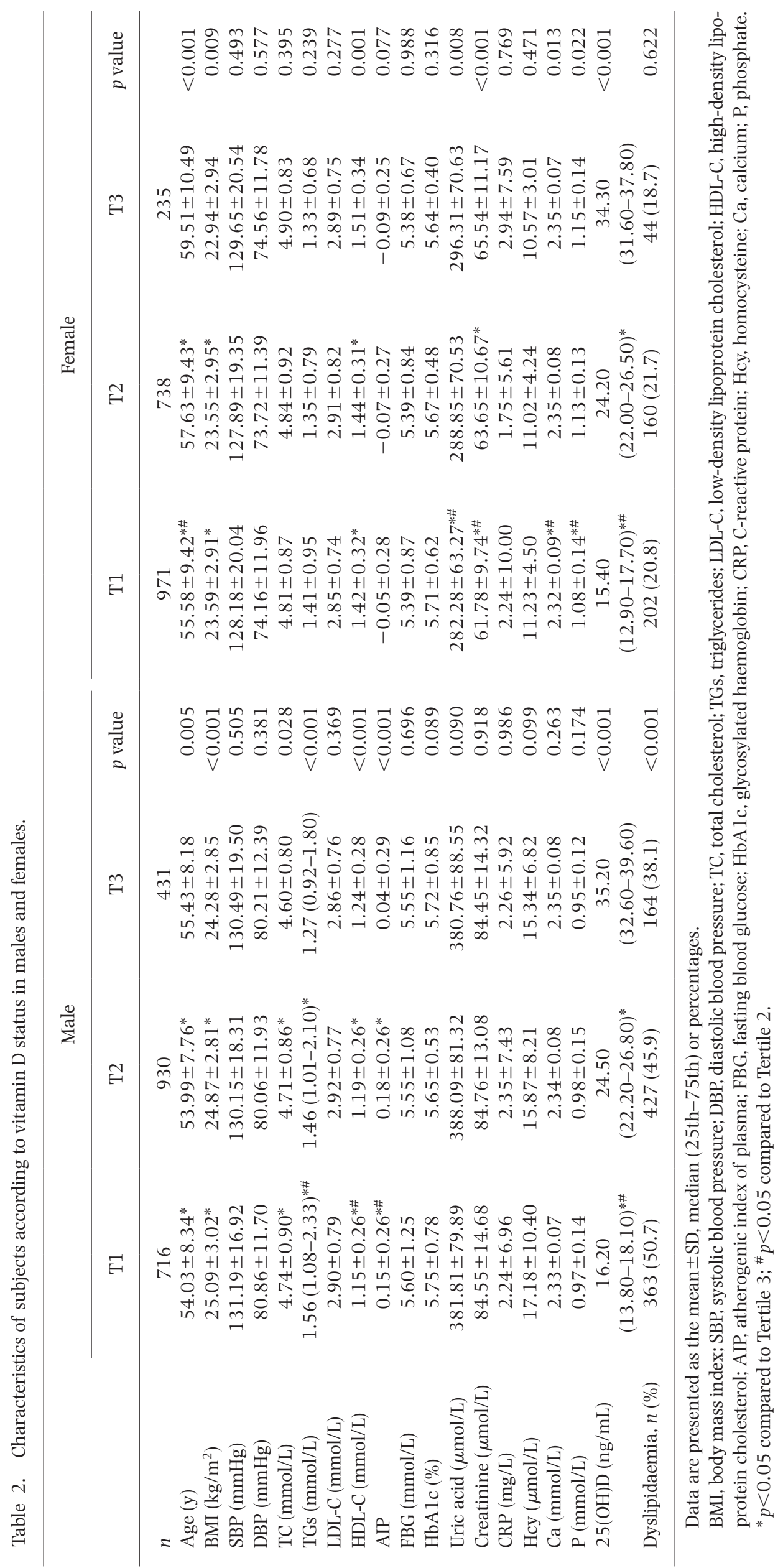


Table 3. Adjusted regression coefficients of vitamin D status with serum lipids and AIP.

\begin{tabular}{lrrrrr}
\hline & \multicolumn{2}{c}{ Model 1 } & & \multicolumn{2}{c}{ Model 2 } \\
\cline { 2 - 3 } \cline { 5 - 6 } & \multicolumn{1}{c}{$\beta$} & $p$ value & & \multicolumn{1}{c}{$\beta$} & $p$ value \\
\hline TC & -0.017 & 0.278 & & -0.020 & 0.219 \\
TGs & -0.077 & $<0.001$ & & -0.063 & $<0.001$ \\
LDL-C & 0.017 & 0.280 & & 0.018 & 0.260 \\
HDL-C & 0.070 & $<0.001$ & & 0.051 & $<0.001$ \\
AIP & -0.085 & $<0.001$ & & -0.068 & $<0.001$ \\
\hline
\end{tabular}

Model 1: adjusted for age, SBP, DBP, FBG, uric acid and creatinine.

Model 2: adjusted for the covariates in Model 1 plus BMI. SBP, systolic blood pressure; DBP, diastolic blood pressure; TC, total cholesterol; TGs, triglycerides; LDL-C, low-density lipoprotein cholesterol; HDL-C, high-density lipoprotein cholesterol; AIP, atherogenic index of plasma; FBG, fasting blood glucose; BMI, body mass index.

The $\beta$ coefficient is a standardized coefficient in multiple linear regression analysis.

25(OH)D and TGs were logarithmically transformed for the analysis.

spectively (all $p$ values $<0.05$ ). In women, 25(OH)D deficiency was associated with an increased prevalence of hypoalphalipoproteinaemia (OR, 2.019; 95\% CI, 1.0443.904; $p=0.037$ ).

We further conducted subgroup analyses stratified by BMI and age in men and women (Supplemental Online Material, Tables S1-S4). There was no evidence of significant interactions between 25(OH)D deficiency, BMI and age on dyslipidaemia in men or evidence of significant interactions between 25(OH)D deficiency and BMI on dyslipidaemia in women (all $p$ values for interactions $>0.05)$. An age-specific interaction was observed in the relationship between serum $25(\mathrm{OH}) \mathrm{D}$ concentrations and the occurrence of hypercholesterolaemia in women $(p=0.036)$.

\section{DISCUSSION}

In this cross-sectional study, we found that vitamin D deficiency was common in a middle-aged and elderly Chinese population and that vitamin D deficiency might be associated with a higher risk for dyslipidaemia after adjusting for potential confounders. Furthermore, hypovitaminosis D is associated with more aggressive dyslipidaemia in males but not in females. In this study population, the mean serum $25(\mathrm{OH}) \mathrm{D}$ concentration was $21.60 \mathrm{ng} / \mathrm{mL}$, and the prevalence of vitamin D deficiency was $42.0 \%$, which was not as notable as the figure reported by Lu et al. regarding a Chinese population (69.2\% deficiency) (14). This deviation could be due to differences in sun exposure, as the participants in our study resided in areas at lower latitudes.

Mounting evidence indicates that vitamin D status may be associated with dyslipidaemia $(11,12)$ and, thus, cardiovascular disease (CVD) $(7,9)$. These studies suggest that serum $25(\mathrm{OH}) \mathrm{D}$ deficiency is associated
Table 4. Adjusted regression coefficients of vitamin D status with serum lipids and AIP in males and females.

\begin{tabular}{lrrrrr}
\hline & \multicolumn{2}{c}{ Model 1 } & & \multicolumn{2}{c}{ Model 2 } \\
\cline { 2 - 3 } \cline { 5 - 6 } & \multicolumn{1}{c}{$\beta$} & \multicolumn{1}{c}{$p$ value } & \multicolumn{1}{c}{$\beta$} & $p$ value \\
\cline { 5 - 6 } Male & & & & \\
TC & -0.046 & 0.033 & & -0.047 & 0.032 \\
TGs & -0.128 & $<0.001$ & & -0.107 & $<0.001$ \\
LDL-C & 0.008 & 0.723 & & 0.008 & 0.706 \\
HDL-C & 0.126 & $<0.001$ & & 0.102 & $<0.001$ \\
AIP & -0.142 & $<0.001$ & & -0.119 & $<0.001$ \\
Female & & & & \\
TC & 0.039 & 0.094 & & 0.035 & 0.127 \\
TGs & -0.025 & 0.257 & & -0.016 & 0.458 \\
LDL-C & 0.034 & 0.143 & & 0.034 & 0.134 \\
HDL-C & 0.077 & 0.001 & & 0.060 & 0.006 \\
AIP & -0.047 & 0.027 & -0.034 & 0.109
\end{tabular}

Model 1: adjusted for age, SBP, DBP, FBG, uric acid and creatinine.

Model 2: adjusted for the covariates in Model 1 plus BMI. SBP, systolic blood pressure; DBP, diastolic blood pressure; TC, total cholesterol; TGs, triglycerides; LDL-C, low-density lipoprotein cholesterol; HDL-C, high-density lipoprotein cholesterol; AIP, atherogenic index of plasma; FBG, fasting blood glucose; BMI, body mass index.

The $\beta$ coefficient is a standardized coefficient in multiple linear regression analysis.

25(OH)D and TGs were logarithmically transformed in males, and 25(OH)D was logarithmically transformed in females for the analysis.

with lower HDL-C levels and higher LDL-C and TG levels $(11,12,23)$. Consistent with these studies, we also found that serum $25(\mathrm{OH}) \mathrm{D}$ concentrations had a strong negative association with TGs and a positive association with HDL-C in all subjects after adjusting for relevant confounders. But our study did not show any significant relationship of serum 25(OH)D with TC or LDL-C levels. However, the results of the relationship between vitamin D and lipid status are conflicting and inconsistent. For example, data from the study by Jorde et al. showed highly significant associations between 25(OH)D and all the lipid parameter in northern Europeans (24). But in a study conducted in American adults, no correlation between 25(OH)D and TGs or HDL-C levels was observed (25). Even in studies conducted in Chinese population, the results are inconsistent. Some studies showed relationships between 25(OH)D with TGs and HDL-C (16, 17), while another study found a relationship with LDL-C (26). The contrasting findings of these studies could be explained by the different characteristics of the study participants.

However, the mechanisms underlying how vitamin D influences the lipid profile remain unclear. One of the possible mechanisms, which was reported in an in vitro study, is the increase in intestinal calcium absorption associated with vitamin D intake. This calcium absorption could then reduce serum TGs by reducing hepatic 


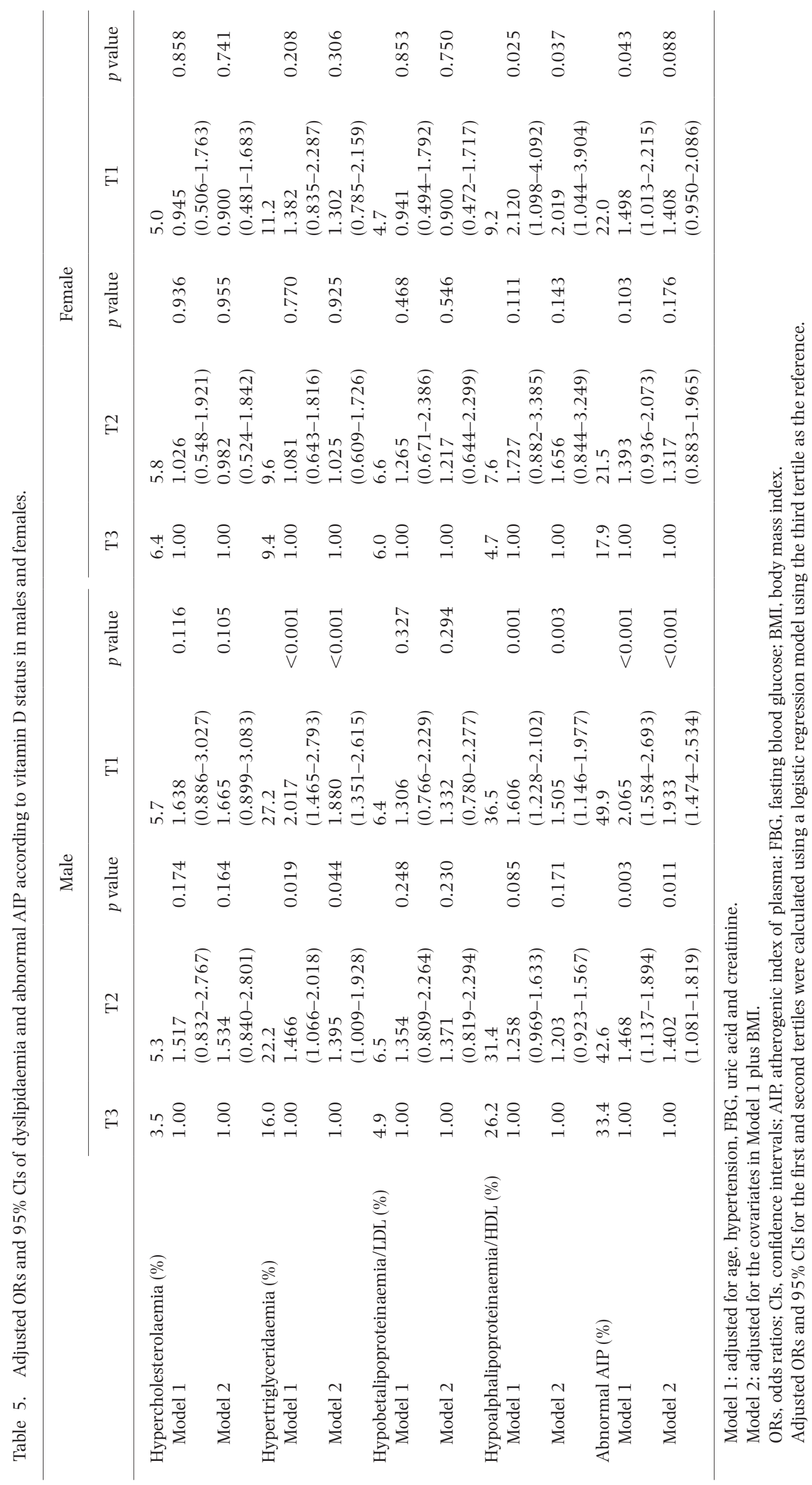


TG synthesis and secretion (27). Moreover, increased levels of intestinal calcium could increase the formation of insoluble calcium-fatty complexes, thus reducing the intestinal absorption of fatty acids (28). Serum levels of TC and LDL-C would be reduced by the decreased absorption of fatty acids. Another possible explanation is that vitamin D has a suppressive effect on the serum parathyroid hormone (PTH) concentration, and a lowered level of PTH may reduce serum TGs by reducing lipolysis (29). In addition, previous studies have suggested that vitamin D deficiency may be associated with impaired $\beta$-cell function and insulin resistance, which could lead to an increase in TG levels and a decrease in HDL-C levels (25). What's more, scientists recently reported a novel mechanism that 25-hydroxyvitamin D might regulate lipid metabolism by inducing degradation of sterol regulatory element-binding protein (SREBP) and SREBP cleavage-activating protein (SCAP), a master regulator of lipogenesis and an escort protein essential for SREBP activation (30).

In the present study, we found that the associations between serum 25(OH)D levels and serum lipids were stronger in men than in women. In men, vitamin D deficiency was associated with a higher risk of hypertriglyceridaemia, hypoalphalipoproteinaemia and abnormal AIP after adjusting for potential confounders. But in women, the association only existed in hypoalphalipoproteinaemia. This finding was in agreement with some previous studies. Yin et al. found that $25(\mathrm{OH}) \mathrm{D}$ was negatively related to TGs and positively related to HDL-C in males and that $25(\mathrm{OH}) \mathrm{D}$ was negatively related to only TGs in females (17). Similarly, Wang et al. reported that $25(\mathrm{OH}) \mathrm{D}$ deficiency was correlated with increased risks of elevated TGs and AIP and reduced HDL-C in men, but the association did not exist in women (16). The mechanisms underlying the sex difference in the association between vitamin $\mathrm{D}$ and lipid profiles have not yet been defined. The difference in hormones and the sensitivity of hormone receptors between the sexes may affect lipid metabolism differently. Many previous studies have suggested that vitamin D concentrations are positively associated with an increase in sexual hormones, such as testosterone in men and oestrogen in women $(31,32)$. As men age, testosterone production decreases gradually, low testosterone has been reported to be associated with greater risks of insulin resistance (33). Therefore, we divided the population into subgroups by age to determine whether there was any difference between serum vitamin D status and lipid profiles. An age-specific interaction was observed in the relationship between serum $25(\mathrm{OH}) \mathrm{D}$ concentrations and the occurrence of hypercholesterolaemia in women $(p=0.036)$. There was a positive relationship between serum $25(\mathrm{OH}) \mathrm{D}$ status and hypercholesterolaemia in women aged $<53$ y (mostly premenopausal participants) but not in women aged $\geqq 53$ y (mostly postmenopausal participants) (Supplemental Online Material, Table S4). It seems that oestrogen may be involved. An interesting finding in this study was the sex disparity. In men, vitamin D deficiency was significantly associated with a higher risk of hyper- triglyceridaemia, hypoalphalipoproteinaemia and abnormal AIP after adjusting for potential confounders. In addition to the above reasons, we imagine that some gene clusters located on the Y chromosome may play an important role, which need to be further identified. Another possible explanation for this difference between men and women is that intestinal calcium absorption may be more vitamin D-dependent in men than in women (34). In addition, lifestyle differences such as physical activity, smoking, alcohol consumption, sun exposure and fat intake could also contribute to the dissimilar results between males and females. However, research on the sex differences in the association between vitamin $\mathrm{D}$ and dyslipidaemia is rare, and well-designed and prospective investigations are needed to obtain more accurate results.

AIP, the logarithmically transformed ratio of TGs to HDL-C, was first described by Dobiásová as a biomarker of plasma atherosclerosis (22). In their later study, AIP was found to be inversely correlated with the diameter of LDL-C particles and to be a surrogate for the smalldense LDL-C level, which is one of the major causative factors of arteriosclerosis and cardiovascular disease (35). In fact, a growing body of research has suggested that AIP is a strong marker for predicting the risk of CVD. In 2010, Onat et al. conducted a prospective study of 2,676 middle-aged adults and reported that AIP is a reliable biomarker for predicting CVD morbidity (18). A large-sample case-control study conducted by Cai et al. indicated that the association of AIP and coronary artery disease also existed in the Chinese population (36). In our study, serum 25(OH)D levels were inversely associated with AIP in men but were not associated with AIP in women, indicating that vitamin D concentrations might be associated with CVD as well.

Our study also has several limitations that merit consideration. First, this was a cross-sectional study, which was unable to estimate cause-effect relationships. Second, other confounding factors that may influence the relationship between vitamin D and lipid status, such as sunlight exposure, dietary vitamin D intake, waist circumference and physical activity, were not assessed. Third, the participants recruited were from a health management centre, and most were from one province. Therefore, the results might not be representative of the whole Chinese middle-aged and elderly population.

Despite these limitations, the present study has several strengths. The major strength is that we collected data from a relatively large sample of a middle-aged and elderly population residing in central China. In addition, we adjusted for many potential covariates, including age, BMI, FBG, uric acid, creatinine, CRP, Hcy, and calcium, making our results more reliable. Furthermore, we also found an association between vitamin $\mathrm{D}$ and AIP, which added to evidence indicating that vitamin D concentrations might be associated with CVD as well.

In summary, our findings showed that vitamin D deficiency was common in a middle-aged and elderly Chinese population and that serum 25(OH)D concentrations were associated with serum lipids. Vitamin D 
deficiency might be associated with a higher risk of dyslipidaemia independent of potential confounders. The relationship between $25(\mathrm{OH}) \mathrm{D}$ and serum lipids was stronger in men than in women. Therefore, vitamin D status may serve as a biomarker for predicting dyslipidaemia or even CVD. Vitamin D status should be taken into consideration, especially in male patients. Further prospective studies with large samples are needed to provide more evidence to confirm the sex-specific relationship between vitamin D and serum lipid profiles. Further gene research may explain the sex disparity.

\section{Author contributions \\ Yongman Lv and Le Wang contributed equally to this work.}

\section{Disclosure of state of COI \\ No conflicts of interest to be declared.}

\section{Acknowledgments}

This study was financially supported by the foundation from the National Natural Science Foundation of China (No. 81873630). We wish to thank all the participants and the staff of the Physical Examination Center of Tongji Hospital for their contributions to this study.

\section{Supporting information}

Supplemental online material is available on J-STAGE.

\section{REFERENCES}

1) Holick MF. 2007. Vitamin D deficiency. $N$ Engl J Med 357: 266-281.

2) El-Khoury JM, Reineks EZ, Wang S. 2011. Progress of liquid chromatography-mass spectrometry in measurement of vitamin D metabolites and analogues. Clin Biochem 44: 66-76.

3) Jensen ME, Ducharme FM, Theoret Y, Belanger AS, Delvin E. 2016. Data in support for the measurement of serum 25-hydroxyvitamin D (250HD) by tandem mass spectrometry. Data Brief 8: 925-929.

4) Chacko SA, Song Y, Manson JE, Van Horn L, Eaton C, Martin LW, McTiernan A, Curb JD, Wylie-Rosett J, Phillips LS. 2011. Serum 25-hydroxyvitamin D concentrations in relation to cardiometabolic risk factors and metabolic syndrome in postmenopausal women. Am J Clin Nutr 94: 209-217.

5) Hewison M. 2010. Vitamin D and the intracrinology of innate immunity. Mol Cell Endocrinol 321: 103-111.

6) Lim S, Kim MJ, Choi SH, Shin CS, Park KS, Jang HC, Billings LK, Meigs JB. 2013. Association of vitamin D deficiency with incidence of type 2 diabetes in high-risk Asian subjects. Am J Clin Nutr 97: 524-530.

7) Perna L, Schoettker B, Holleczek B, Brenner H. 2013. Serum 25-hydroxyvitamin D and incidence of fatal and nonfatal cardiovascular events: a prospective study with repeated measurements. J Clin Endocrinol Metab 98: 4908-4915.

8) Lavie CJ, Dinicolantonio JJ, Milani RV, O'Keefe JH. 2013. Vitamin D and cardiovascular health. Circulation 128: 2404-2406.

9) Wang TJ, Pencina MJ, Booth SL, Jacques PF, Ingelsson E, Lanier K, Benjamin EJ, D'Agostino RB, Wolf M, Vasan RS. 2008. Vitamin D deficiency and risk of cardiovascu- lar disease. Circulation 117: 503-511.

10) Gu DF, Gupta A, Muntner P, Hu SS, Duan XF, Chen JC, Reynolds RF, Whelton PK, He J. 2005. Prevalence of cardiovascular disease risk factor clustering among the adult population of china-Results from the International Collaborative Study of Cardiovascular Disease in Asia (InterAsia). Circulation 112: 658-665.

11) Jorde R, Grimnes G. 2011. Vitamin D and metabolic health with special reference to the effect of vitamin D on serum lipids. Prog Lipid Res 50: 303-312.

12) Lupton JR, Faridi KF, Martin SS, Sharma S, Kulkarni K, Jones SR, Michos ED. 2016. Deficient serum 25-hydroxyvitamin D is associated with an atherogenic lipid profile: The Very Large Database of Lipids (VLDL-3) study. J Clin Lipidol 10: 72-81 e71.

13) Karhapaa P, Pihlajamaki J, Porsti I, Kastarinen M, Mustonen J, Niemela O, Kuusisto J. 2010. Diverse associations of 25-hydroxyvitamin D and 1,25-dihydroxy-vitamin D with dyslipidaemias. J Intern Med 268: 604-610.

14) Lu L, Yu Z, Pan A, Hu FB, Franco OH, Li H, Li X, Yang X, Chen Y, Lin X. 2009. Plasma 25-hydroxyvitamin D concentration and metabolic syndrome among middleaged and elderly Chinese individuals. Diabetes Care 32: 1278-1283.

15) Chon SJ, Yun BH, Jung YS, Cho SH, Choi YS, Kim SY, Lee BS, Seo SK. 2014. Association between vitamin D status and risk of metabolic syndrome among Korean postmenopausal women. Plos One 9: e89721.

16) Wang Y, Si S, Liu J, Wang Z, Jia H, Feng K, Sun L, Song SJ. 2016. The associations of serum lipids with vitamin D status. PLoS One 11: e0165157.

17) Yin X, Sun Q, Zhang X, Lu Y, Sun C, Cui Y, Wang S. 2012. Serum $25(\mathrm{OH}) \mathrm{D}$ is inversely associated with metabolic syndrome risk profile among urban middle-aged Chinese population. Nutr J 11: 68.

18) Onat A, Can G, Kaya H, Hergenc G. 2010. "Atherogenic index of plasma" $(\log (10)$ triglyceride/high-density lipoprotein-cholesterol) predicts high blood pressure, diabetes, and vascular events. J Clin Lipidol 4: 89-98.

19) Shen S, Lu Y, Qi H, Li F, Shen Z, Wu L, Yang C, Wang L, Shui K, Wang Y. 2016. Association between ideal cardiovascular health and the atherogenic index of plasma. Medicine (Baltimore) 95: e3866.

20) Holick MF, Binkley NC, Bischoff-Ferrari HA, Gordon CM, Hanley DA, Heaney RP, Murad MH, Weaver CM. 2011. Evaluation, treatment, and prevention of vitamin D deficiency: an Endocrine Society clinical practice guideline. J Clin Endocrinol Metab 96: 1911-1930.

21) Jellinger PS, Smith DA, Mehta AE, Ganda O, Handelsman Y, Rodbard HW, Shepherd MD, Seibel JA; AACE Task Force for Management of Dyslipidemia and Prevention of Atherosclerosis. 2012. American Association of Clinical Endocrinologists' Guidelines for management of dyslipidemia and prevention of atherosclerosis. Endocr Pract 18 (Suppl 1): 1-78.

22) Dobiásová M. 2006. AIP-atherogenic index of plasma as a significant predictor of cardiovascular risk: from research to practice. Vnitr Lek 52: 64-71.

23) Sun $\mathrm{X}$, Cao Z-B, Tanisawa K, Ito T, Oshima S, Ishimi $Y$, Tabata I, Higuchi M. 2015. Associations between the serum 25(OH)D concentration and lipid profiles in Japanese men. J Atheroscler Thromb 22: 355-362.

24) Jorde R, Figenschau Y, Hutchinson M, Emaus N, Grimnes G. 2010. High serum 25-hydroxyvitamin D concentrations are associated with a favorable serum lipid profile. 
Eur J Clin Nutr 64: 1457-1464.

25) Chiu KC, Chu A, Go VLW, Saad MF. 2004. Hypovitaminosis D is associated with insulin resistance and beta cell dysfunction. Am J Clin Nutr 79: 820-825.

26) Zhang MC, Li HX, Liu HM, Lei H, Han L, Gao M, Mao JF, Xu XJ. 2014. Serum vitamin D is low and inversely associated with LDL cholesterol in the Kazak ethnic population: a cross-sectional study. Med Sci Monit 20: 1274-1283.

27) Zittermann A, Frisch S, Berthold HK, Goetting C, Kuhn J, Kleesiek K, Stehle P, Koertke H, Koerfer R. 2009. Vitamin D supplementation enhances the beneficial effects of weight loss on cardiovascular disease risk markers. Am J Clin Nutr 89: 1321-1327.

28) Christensen R, Lorenzen JK, Svith CR, Bartels EM, Melanson EL, Saris WH, Tremblay A, Astrup A. 2009. Effect of calcium from dairy and dietary supplements on faecal fat excretion: a meta-analysis of randomized controlled trials. Obes Rev 10: 475-486.

29) Zemel MB, Shi H, Greer B, Dirienzo D, Zemel PC. 2000. Regulation of adiposity by dietary calcium. FASEB J 14: 1132-1138.

30) Asano L, Watanabe M, Ryoden Y, Usuda K, Yamaguchi T, Khambu B, Takashima M, Sato S, Sakai J, Nagasawa K. 2017. Vitamin D metabolite, 25-hydroxyvitamin D, regulates lipid metabolism by inducing degradation of SREBP/SCAP. Cell Chem Biol 24: 207-217.
31) Parikh G, Varadinova M, Suwandhi P, Araki T, Rosenwaks Z, Poretsky L, Seto-Young D. 2010. Vitamin D regulates steroidogenesis and insulin-like growth factor binding protein-1 (IGFBP-1) production in human ovarian cells. Horm Metab Res 42: 754-757.

32) Rafiq R, van Schoor NM, Sohl E, Zillikens MC, Oosterwerff MM, Schaap L, Lips P, de Jongh RT. 2016. Associations of vitamin D status and vitamin D-related polymorphisms with sex hormones in older men. J Steroid Biochem Mol Biol 164: 11-17.

33) Li J, Lai H, Chen S, Zhu H, Lai S. 2017. Interaction of sex steroid hormones and obesity on insulin resistance and type 2 diabetes in men: the Third National Health and Nutrition Examination Survey. J Diabetes Complications 31: 318-327.

34) Morris ME, Lee HJ, Predko LM. 2003. Gender differences in the membrane transport of endogenous and exogenous compounds. Pharmacol Rev 55: 229-240.

35) Dobiasova M, Frohlich J. 2001. The plasma parameter $\log (\mathrm{TG} / \mathrm{HDL}-\mathrm{C})$ as an atherogenic index: correlation with lipoprotein particle size and esterification rate in apoB-lipoprotein-depleted plasma (FERHDL). Clin Biochem 34: 583-588.

36) Cai G, Shi G, Xue S, Lu W. 2017. The atherogenic index of plasma is a strong and independent predictor for coronary artery disease in the Chinese Han population. Medicine (Baltimore) 96: e8058. 